1 A Comparison of Measured Room Acoustics Metrics Using a Spherical Microphone Array and Conventional

2 Methods

3 David A. Dick and Michelle C. Vigeant

4 Graduate Program in Acoustics, The Pennsylvania State University, University Park, PA 16802, USA,

$5 \quad$ Vigeant@engr.psu.edu, +1 814-863-7145

6 Key words: spherical microphone array; room acoustics; spatial measures; measurement hardware;

7 room impulse response

8

9 NOTE: Portions of this work were presented at two conferences:

10 - Dick, D.A., and Vigeant, M.C., "Measurement repeatability of late lateral energy level and lateral 11 energy fraction." J. Acoust. Soc. Am., 135:2236, 2014 [Abstract only]

12 - Dick, D.A., and Vigeant, M.C., “A Comparison of Late Lateral Energy (GLL) and Lateral Energy 13 Fraction (LF) Measurements Using a Spherical Microphone Array and Conventional Methods." 14 Proc. of the EAA Joint Symposium on Auralization and Ambisonics, Berlin, 2014 [Proceeding paper, 7 pages] 


\section{A Comparison of Measured Room Acoustics Metrics Using a Spherical Microphone Array and Conventional Methods} The traditional microphone configuration used to measure room impulse responses (IRs) according to ISO 3382:2009 is an omnidirectional and figure-8 microphone pair. IRs measurements were taken in a 2500seat auditorium to determine how the results from a spherical microphone array (an mh acoustics Eigenmike-em32) compare to those from the traditional microphone setup (a Brüel \& Kjær Type-4192 omnidirectional microphone and a Sennheiser MKH30 figure-8 microphone). Measurements were obtained at six receiver locations, with three repetitions each in order to first evaluate repeatability. The metrics considered in this study were: reverberation time (T30), early decay time (EDT), clarity index (C80), strength $(G)$, lateral energy fraction $\left(\mathrm{J}_{\mathrm{LF}}\right)$ and late lateral energy level $\left(\mathrm{L}_{J}\right)$. Before calculating these quantities, the IRs were filtered to equalize the frequency response of the microphones and sound source. For the spherical array measurements, the omnidirectional (monopole) and figure-8 (dipole) patterns were extracted using beamforming. In terms of repeatability, the average standard deviation of the three measurements at each receiver location averaged across all metrics, receivers, and octave bands was found to be 0.01 just noticeable differences (JNDs). The analysis comparing the measurements from the two microphone configurations yielded differences which were less than 1 JND for the majority of metrics, with a few exceptions of EDT and C80 slightly above 1 JND. Based on this case study, these results indicate that spherical microphone arrays can be used to obtain valid room IR measurements, which will allow for the development of new metrics utilizing the higher spatial resolution made possible with spherical arrays.

\section{INTRODUCTION}

Spherical microphone arrays contain a number of microphones arranged on the surface of a compact sphere and can be used to obtain spatial information about sound fields. The spherical configuration of the array enables a convenient way to beamform directional patterns in any direction in 3D space using a spatial Fourier transform and processing the signals in the spherical harmonics domain [1] [2]. In recent years, spherical microphone arrays have begun to be utilized in room acoustics applications to analyze the directional properties of reverberant spaces [3-6]. Room impulse responses (IRs) measured with spherical arrays have been analyzed to determine the direction of arrival of early reflections in rooms $[3,4,5]$. A recent study also evaluated IR measurements obtained in performing arts spaces using a 16-channel spherical microphone array by beamforming the IRs in the azimuthal plane and comparing different audience receiver positions [6].

Previous room acoustics studies involving spherical microphone arrays have not included analyses of the IRs to calculate established room acoustics metrics as defined in Annex A of ISO 3382 [7]. These metrics require measurements made using a pair of microphones, one with an omnidirectional directivity pattern and a second one with a figure-of-eight (figure-8) directivity pattern. Alternatively, these directivity patterns can be obtained from spherical microphone array measurements by extracting the zeroth (monopole) and first order (dipole) spherical harmonic components, respectively. Before this analysis can be done, however, room acoustics metrics using spherical microphone arrays must be verified against traditional methods in order to gain confidence that the measurements are consistent. This research is especially necessary due to the fact that previous work has shown a large variation in measured parameters made between different microphone types and with different measurement teams [20-25]. Additionally, this comparison is necessary since spherical microphone arrays are generally larger than conventional measurement microphones, and therefore may alter the sound field if the sound wave that is scattered from the array reflects off of nearby objects and returns to the microphone array [1]. 
Obtaining room acoustics metrics with spherical microphone arrays may offer some advantages compared to measurements made with conventional microphones. Spatial measures are typically obtained using a figure-8 microphone. Commercially available figure-8 microphones are not laboratory-grade and may not have ideal directivity, frequency response, or linearity; whereas spherical array microphones are typically constructed using laboratory-grade microphone capsules. Spherical microphone arrays also enable the researcher to rotate the figure- 8 pattern in post-processing to perfectly align the pattern to the source, which could reduce measurement uncertainty. Finally, current spherical microphone array technology enables beamforming utilizing spherical harmonics up to third- or fourth-order, which can be used to create new room acoustics metrics with a much higher spatial resolution than the traditional first-order 72 dipole.

The purpose of this case study was to compare measurements taken in accordance with the ISO 3382 standard using a traditional omnidirectional and figure-8 microphone pair with measurements taken using a spherical microphone array. This comparison is required in order to gain confidence that room acoustics measurements made with a spherical microphone array can be directly compared to measurements made with traditional methods. Once this verification is complete, new metrics with higher spatial resolution can be developed.

\subsection{Room Acoustics Metrics}

The metrics that were evaluated in this study are defined in ISO 3382 and accompanying Annex A. The omnidirectional measures are reverberation time (T30), measured from a $30 \mathrm{~dB}$ decay from the Schroeder backwards integrated curve; early decay time (EDT), measured from the slope of the first $10 \mathrm{~dB}$ decay of the Schroeder backwards integrated curve; clarity index (C80), the ratio of the early sound in the first 80 ms to the late sound; and strength (G), the energy in the room IR normalized to the level of the sound source measured at a distance of $10 \mathrm{~m}$ in a free field. In addition to the commonly used omnidirectional measures, metrics used to predict the spatial impression of a room are included in Annex A of ISO 3382. Spatial impression is one characteristic that has been shown to be related to overall room impression [8, $9,10]$. Previous research proposed that spatial impression should be formally divided into two distinct components [11]: the apparent source width (ASW) as being associated with the early lateral reflections, and listener envelopment (LEV), which is related to late lateral reflections [12]. A number of objective measures have been proposed to predict both ASW and LEV that utilize either directional microphones or a binaural head [13]. The two spatial metrics that have gained the largest acceptance in the architectural acoustics community are early lateral energy fraction $\left(U_{L F}\right.$, previously $\left.L F\right)$ [14], which is used to predict ASW, and late lateral energy level ( $L_{J}$, previously $G L L, L G$, and $\left.L G_{80}^{\infty}\right)$ [12], which is used to predict LEV. Both of these metrics are included in ISO 3382 Annex A and were evaluated as part of this study. $J_{L F}$ is the ratio of early lateral energy to total early energy:

$$
J_{L F}=\frac{\int_{5 m s}^{80 m s} p_{f}^{2}(t) d t}{\int_{0}^{80 m s} p_{o}^{2}(t) d t}
$$

where $p_{f}(t)$ is the IR measured with a figure-8 microphone, and $p_{o}(t)$ is the IR measured with an omnidirectional microphone. $L_{J}$ is the ratio of the late lateral energy to the normalized source energy:

$$
L_{J}=10 \log \left[\frac{\int_{80 m s}^{\infty} p_{f}^{2}(t) d t}{\int_{0}^{\infty} p_{10}^{2}(t) d t}\right][\mathrm{dB}],
$$


where $p_{10}(t)$ is the IR of the sound source normalized at a distance of $10 \mathrm{~m}$ away in a free field.

\section{MEASUREMENT UNCERTAINTY}

102

A number of studies have shown that there is a high degree of measurement uncertainty in room acoustics metrics obtained from room IRs [15-25]. Specific sources of uncertainty and studies between measurement teams are summarized below. A common method to evaluate uncertainty is to compare measurements in terms of just noticeable differences (JNDs). The JND for each room acoustics parameter is included in the Annex A of ISO 3382 [7]: 5\% for T30 and EDT, $1 \mathrm{~dB}$ for C80, $1 \mathrm{~dB}$ for G, 0.05 for JL, 0.05 for definition (D), and $10 \mathrm{~ms}$ for center time (Ts); the JND for $L_{J}$ is not known. For the purposes of this study, the JND for $G$ will be used for $L$.

The contributions of different sources of uncertainty to the overall measurement uncertainty has been studied in Ref. [15]. The main contributions to measurement uncertainty are source position and orientation, microphone placement and orientation, source directivity, microphone directivity, and measurement hardware frequency response. Source directivity, in particular, has been shown to be a significant portion of the measurement uncertainty as a result of non-uniform source directivity. The most common sound sources used in room acoustics are dodecahedron loudspeakers, which typically become directional above approximately $1 \mathrm{kHz}$. Therefore, the orientation of the source can yield different results in room acoustic metrics $[16,17]$. A second major contributor is microphone placement, where measures can vary widely even within a single seat location $[18,19]$. Additional sources of uncertainty include ambient room conditions (i.e. temperature and humidity), evaluation methods (e.g. different signal processing and filtering methods), room noise, and equipment noise.

Studies comparing metrics calculated from IRs obtained from different measurement teams show differences that exceed the JND of each metric in most cases [20-22]. One of the earliest studies comparing the results from four measurement teams showed that the standard deviation across the teams were around $5 \%$ to $10 \%$ for T30, EDT, D, and $\mathrm{T}_{\mathrm{s}}$, and around $0.5 \mathrm{~dB}$ for $\mathrm{C} 80$ and $\mathrm{G}$ from $1 \mathrm{kHz}$ to 4 $\mathrm{kHz}$, which are all on the order of 1-2 JNDs [20]. The largest differences tended to occur in the $125 \mathrm{~Hz}$ octave band. Additionally, larger differences were found in LF measurements with differences up to 4 JNDs at $1 \mathrm{kHz}$.

The first phase of the third round robin on room acoustics simulation programs was to collect measurement data on the space that was to be modeled [21]. T30, EDT, C80, and G measurements all showed differences well above 1 JND with the largest differences in the $125 \mathrm{~Hz}$ octave band. Again, the largest differences were found in the parameter $L_{F}$, which were on the order of 3 to 5 JNDs in various octave bands and receiver positions. As part of the third round robin study, some follow-up measurements using three figure-8 microphones of the same make and model (Neumann KM86) revealed significant differences in measurements taken with the microphones at different orientations (i.e. rotated 180 degrees). One possible source of this measurement error was hypothesized to be due to changes in the microphone sensitivity of each diaphragm due to aging.

A third study compared measurements made in opera houses using different measurement hardware and excitation techniques [22]. Differences were found using different types of sources, e.g. different types of dodecahedron loudspeakers and impulsive sources such as balloons or starter pistols. Measurements were compared using an omnidirectional microphone and two sets of binaural microphones in the same 
dummy head. The two binaural microphones had differences in $\mathrm{C} 80$ up to $0.5 \mathrm{~dB}$ at low frequencies, which is below $1 \mathrm{JND}$. Measurements made comparing the binaural microphones to the omnidirectional microphone showed differences greater than $1 \mathrm{~dB}$ for C80 (1 JND) and 15\% for reverberation time (3 JNDs).

A handful of studies have been conducted in which the same measurement team evaluated measurements using different microphones. In these studies, measurement uncertainty caused by the source was significantly reduced because researchers used the same source for all of the measurements. Additionally, uncertainty due to microphone placement should have also been reduced since placement within a team is likely to be more consistent than across teams. Therefore, in these studies a majority of the uncertainty should be due to the differences in microphone response. A study in 2006 compared parameter differences for measurements made with four different types of microphones [23]. Standard deviations for the 10 measurements made with each microphone type were small, indicating that measurements were consistent and repeatable, but differences between microphone types were found to be approximately 1 JND for T20 and G, and between 1 and 2 JNDs for LF. A second study compared the results of spatial measurements made from a figure- 8 and omnidirectional microphone pair and an intensity probe made with two omnidirectional capsules [24]. Differences in $\mathrm{J}_{\mathrm{LF}}$ between the two microphone types were between 1 to 4 JNDs in various octave bands. The authors of the study speculated that most of the differences in the measurements were due the directivity of the microphones, which were found to deviate from the ideal patterns. A more recent case study was conducted to further evaluate the measurement uncertainty of the spatial measures $L_{J}$ and $J_{L F}$ in terms of microphone orientation, spacing between the microphone pair, and microphone type [25]. A total of five different makes and models of figure- 8 microphones were evaluated by taking measurements in a small lecture hall with about 100 seats. The average differences due to microphone spacing, which varied between 64 to $152 \mathrm{~mm}$, and microphone orientation, were found to be relatively small for $L_{J}$, and $J_{L F}$, with differences below $1 \mathrm{JND}, 0.2 \mathrm{~dB}$ and 0.02 , respectively. On the other hand, the effect of microphone type was more significant for $L_{J}$, with variations on the order of $1.5 \mathrm{~dB}$, but approximately $1 \mathrm{JND}$ for $\mathrm{J}_{\mathrm{LF}}$.

Based on findings from the aforementioned studies, the goal of ensuring differences in microphone types that are under 1 JND may be difficult to achieve in practice. In most of the studies involving measurement uncertainty, measurements made in the same location with different microphones or between different microphone teams exceeded 1 JND, which would indicate a perceptible difference between the measurements even though there may be none. In general, findings show that there is more uncertainty associated with spatial measures using a figure- 8 microphone than in metrics only involving omnidirectional microphone measurements [20-25].

\section{SPHERICAL MICROPHONE ARRAY PROCESSING AND BEAMFORMING}

As shown in [26], the pressure on the surface of a sphere due to an incident plane can be represented as an infinite sum of spherical harmonics:

$$
p(r, \vartheta, \varphi, t)=P_{0} 4 \pi \sum_{n=0}^{\infty} i^{n} b_{n}(k a) \sum_{m=-n}^{n} Y_{n}^{m}(\vartheta, \varphi) Y_{n}^{m *}\left(\vartheta_{i}, \varphi_{i}\right) e^{i \omega t},
$$


where $p$ is the total sound pressure, $P_{0}$ is the pressure amplitude, $i$ is the imaginary number $\sqrt{-1}, \vartheta$ is the elevation angle, $\varphi$ is the azimuthal angle, and the direction of the incident wave is $\left(\vartheta_{i}, \varphi_{i}\right)$, and $*$ denotes the complex conjugate. $Y_{n}^{m}$ are the spherical harmonics of order $n$ and degree $m$, which are defined as:

$$
Y_{n}^{m}(\vartheta, \varphi)=\sqrt{\frac{(2 n+1)}{4 \pi} \frac{(n-m) !}{(n+m) !}} P_{n}^{m}(\cos \vartheta) e^{i m \varphi} .
$$

The coefficients $b_{n}$ are known as the plane wave modal coefficients and are dependent on the array geometry and the boundary conditions at the surface of the sphere. For a rigid sphere, the coefficients are [2]:

$$
b_{n}(k a)=j_{n}(k a)-\frac{j_{n}{ }^{\prime}(k a)}{h_{n}^{(2)^{\prime}}(k a)} h_{n}^{(2)}(k a),
$$

where $j_{n}$ are spherical Bessel functions of order $n, h_{n}^{(2)}$ are spherical Hankel functions of the second kind 183 of order $n$, and 'signifies a derivative with respect to the argument.

The spherical harmonics form an orthonormal basis set, meeting the orthogonality condition:

$$
\int Y_{n \prime}^{m \prime}(\vartheta, \varphi) Y_{n}^{m *}(\vartheta, \varphi)=\delta_{n n^{\prime}} \delta_{m m^{\prime}}
$$

where $\delta$ is the Kronecker delta function. Because of the orthogonality property of the spherical harmonics, the spatial Fourier coefficients for the spherical harmonics $\widetilde{P}_{n m}(k a)$ can be obtained by applying weights to each microphone signal and summing the signals together:

$$
\tilde{P}_{n m}(k a)=\frac{1}{b_{n}(k a)} \sum_{s=1}^{S} P_{s}(k a) Y_{n}^{m *}\left(\vartheta_{s}, \varphi_{s}\right),
$$

where $P_{S}$ is the complex pressure in the frequency domain measured at microphone $s$, obtained by taking a discrete Fourier transform (DFT) of each microphone signal, and $\left(\vartheta_{s}, \varphi_{s}\right)$ is the location of the microphone on the sphere.

The spatial Fourier components can be weighted and combined to beamform different directional patterns in the spherical harmonics domain. A plane wave decomposition can be performed by summing the components together and setting the beamforming weights to unity [27]:

$$
P\left(\vartheta_{l}, \varphi_{l}\right)=\sum_{n=0}^{N} \sum_{m=-n}^{n} \tilde{P}_{n m}(k a) Y_{n}^{m}\left(\vartheta_{l}, \varphi_{l}\right),
$$

where $\left(\theta_{l}, \phi_{l}\right)$ is the look direction of the beam (the direction in which the main lobe of the beam pattern is oriented). To calculate the parameters in ISO 3382 [7], the omnidirectional (monopole) and figure-8 (dipole) components must be extracted from spatial Fourier coefficients. The omnidirectional component 197 


$$
P_{o}=Y_{0}^{0} \tilde{P}_{00}=\frac{1}{(4 \pi)^{2} b_{0}(k a)} \sum_{S=1}^{S} P_{S}(k a)
$$

The figure- 8 component can be extracted by weighting and summing the first order spherical harmonic components:

$$
\begin{aligned}
P_{f}=\sum_{m=-1}^{1} \tilde{P}_{1 m} Y_{1}^{m}\left(\theta_{l}, \phi_{l}\right) & \\
= & \frac{1}{b_{1}(k a)} \sum_{m=-1}^{1} Y_{1}^{m}\left(\theta_{l}, \phi_{l}\right) \sum_{s=1}^{S} P_{s}(k a) Y_{1}^{m *}\left(\vartheta_{s}, \varphi_{s}\right),
\end{aligned}
$$

where $\left(\theta_{l}, \phi_{l}\right)$ is the look direction of the beam (where the dipole is a maximum). The dipole pattern must be steered in the proper direction to calculate room acoustics parameters. The look direction should be chosen such that the elevation angle is pointed toward the source, and the azimuthal angle is the direction of the source plus 90 degrees. This process will align the null plane of the dipole pattern with the sound source. The time domain omnidirectional and figure- 8 signals are calculated using an inverse DFT of $P_{o}$ and $P_{f}$, respectively. Room acoustics metrics can then be calculated using the beamformed IRs.

The theoretical weights used in the beamforming analysis, given in Eqns. 7-10, assume that the spherical microphone array used in this study is close to ideal, i.e. the geometry is a uniformly sampled rigid sphere and the array elements are perfectly matched in magnitude and phase. The theoretical weights were found to work well in this application and the desired directivity patterns were achieved (see Section 4.2). However, any phase mismatches or calibration differences can cause a degradation in the achieved beampattern. If mismatches between microphone capsules are present, then it may be advantageous to use weights based on measurements of the array rather than theoretical weights [28] [29]. Applying this technique can improve the performance of the beamformer in order to achieve a more ideal directivity pattern.

\section{MEASUREMENTS}

\section{$216 \quad 4.1$ Measurement Hardware}

217 The spherical microphone array used in this study was an Eigenmike em32 array made by mh Acoustics, 218 as shown in Figure 1a [30]. This array consists of 32 omnidirectional electret microphone capsules distributed uniformly on an 8.4-cm diameter rigid sphere. The microphone capsules are individually calibrated to account for magnitude differences between capsules. The upper frequency limit of the spherical array due to spatial aliasing is approximately $8 \mathrm{kHz}$. The Eigenmike system includes the Eigenmike Interface Box (EMIB), which is used to convert the Eigenmike data into a standard Firewire interface that can be controlled via an ASIO driver. The EMIB also contains a digital output, which allows the EMIB to send the excitation signals to the sound source. The ASIO driver and digital output make it possible to take IR measurements with synchronized inputs and outputs using commercially available room acoustics measurement software. This system allows for simultaneous measurements of the 32 individual microphone channels of the Eigenmike.

Traditional IR measurements were made using a Brüel \& Kjær (B\&K) Type 419212.7 mm (0.5 inch) omnidirectional microphone and a Sennheiser MKH 30 figure-8 microphone. These microphones were 
chosen because they are representative of a typical measurement microphone and studio-grade figure-8 microphone, respectively. The omnidirectional and figure- 8 microphones were placed on the same microphone stand $7.6 \mathrm{~cm}$ apart from each other and measured simultaneously, as shown in Figure $1 \mathrm{~b}$. This spacing was chosen to allow for the microphones to be adequately far enough apart so as to minimize the effects on the other microphone, but close enough so that they were measuring approximately the same point in space [25].

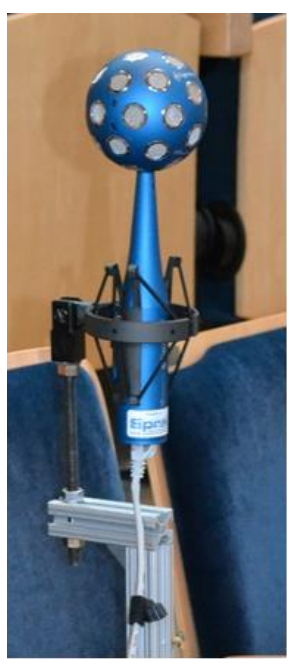

(a)

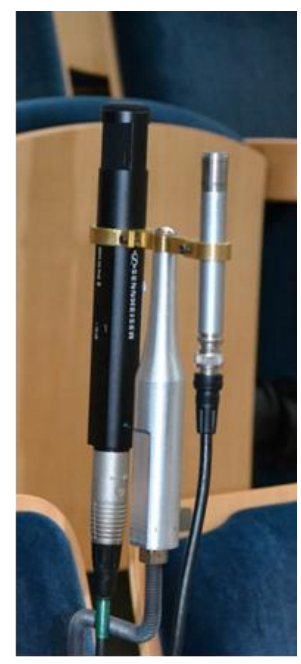

(b)

Figure 1: Microphones used in this study. (a) Eigenmike em32 spherical microphone array and (b) a Brüel \& Kjær (B\&K) Type 4192 omnidirectional and Sennheiser MKH3O figure-8 microphone pair. 

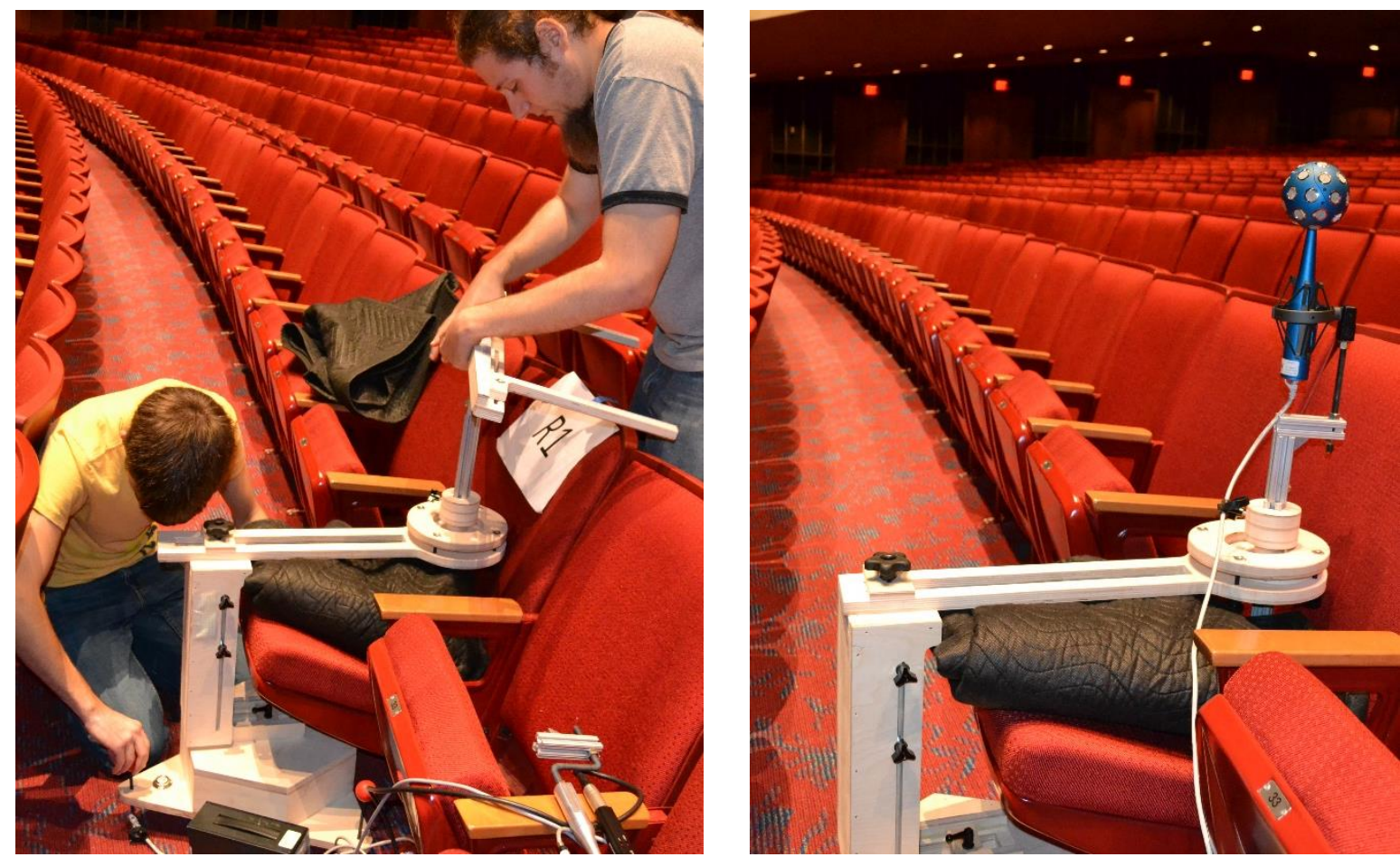

Figure 2: Custom microphone stand used for accurate and precise placement of microphones. The photo on the left shows the stand being used to place the microphone and the one on the right shows the microphone in the final position.

\subsection{Anechoic Chamber Directivity and Frequency Response Measurements}

Dodecahedron loudspeakers that are commonly used for room acoustics measurements are typically about $40-50 \mathrm{~cm}$ in diameter, which limits the omnidirectional radiation up to approximately $1 \mathrm{kHz}$. Consequently, ISO 3382:2009 requires the measurement of the sound source as a function of azimuthal angle in a free field to calculate certain metrics from the IR, specifically $G$ and $L$, [7]. IR measurements were made in an anechoic chamber at 12.5 degree increments around the B\&K loudspeaker at a distance of $3.55 \mathrm{~m}$ from the source using a B\&K $419112.7 \mathrm{~mm}$ (0.5 inch) free-field microphone as specified in the standard to account for the variation in directivity of the source as a function of azimuthal angle. The 29 measurements were energy-averaged in the frequency domain and normalized to a distance of $10 \mathrm{~m}$ from the source. The averaged result was used as the normalization IR for $G$ and $L_{\text {J. }}$. The averaged result was also inverted and used to generate a filter to equalize the frequency response of the loudspeaker.

Anechoic measurements were also conducted to evaluate the sensitivity, frequency response, and directivity of both the Eigenmike array and the Sennheiser MKH30. The sensitivity was measured by playing a $1 \mathrm{kHz}$ tone over the dodecahedron loudspeaker and measuring it with both microphones and a calibrated sound analyzer, B\&K type 2250 . The directivities were evaluated by measuring the IR of the microphones as a function of angle with a stationary loudspeaker placed $2 \mathrm{~m}$ from the microphone. The IRs of the microphones were measured every 3 degrees. The Sennheiser MKH30 and spherical array beamformed dipoles both have the correct directivity pattern with two clear nulls and a matching response at both maxima, as shown in Figure 3. 

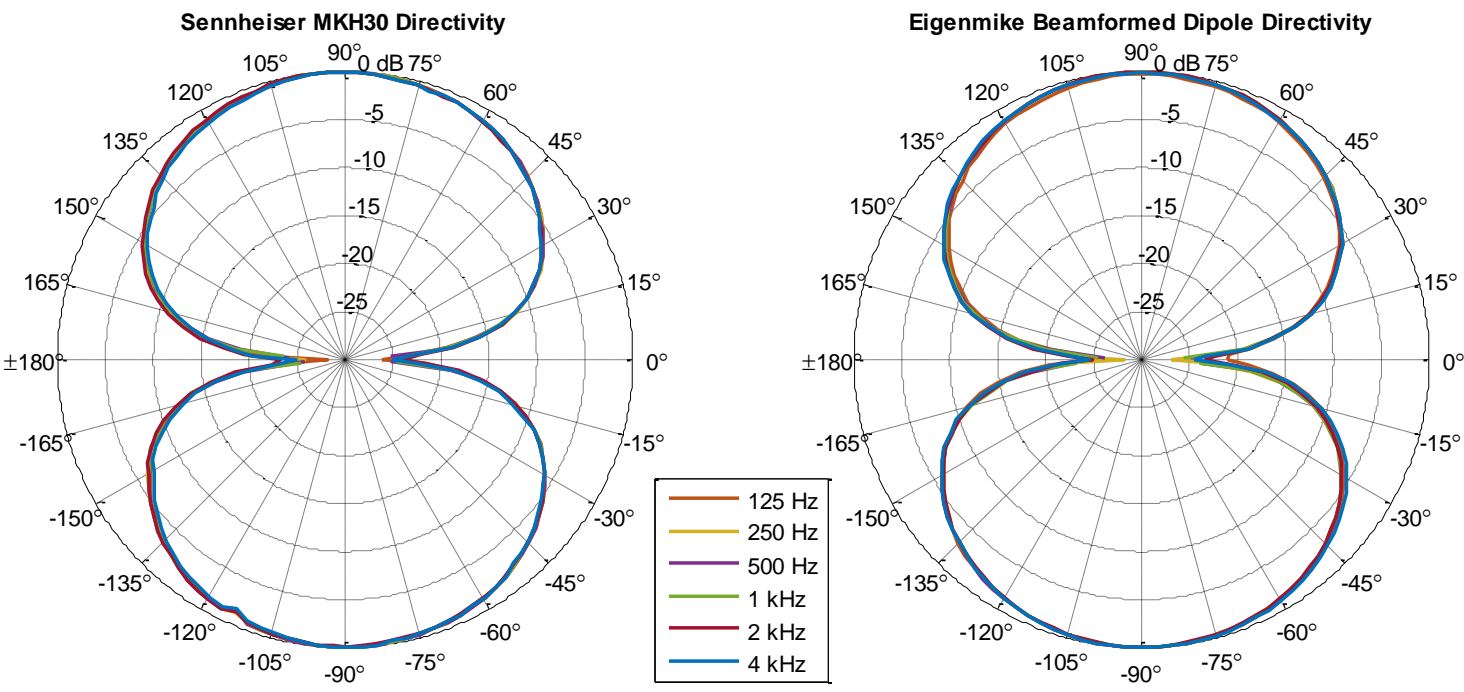

Figure 3: Microphone directivity plots for the Sennheiser MKH3O figure-8 microphone (left) and Eigenmike em32 beamformed dipole pattern (right).

The deviation of the microphone directivity patterns from the ideal patterns for the Sennheiser MKH30 and Eigenmike em32 microphones are shown in Figure $4 a$ and $4 b$, respectively. For the two figure-8 patterns, the deviations are a fraction of $1 \mathrm{~dB}$, with the exception of angles close to the nulls, where the majority of the sound is being rejected but the rejection is not infinite. Additionally, the directivity pattern for the Eigenmike em 32 beamformed omnidirectional pattern is very close to ideal (Fig. 4c) with average deviations also on the order of a fraction of $1 \mathrm{~dB}$.

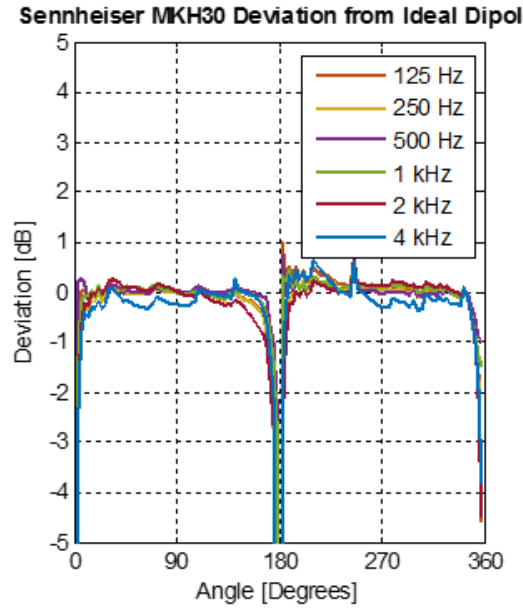

(a)

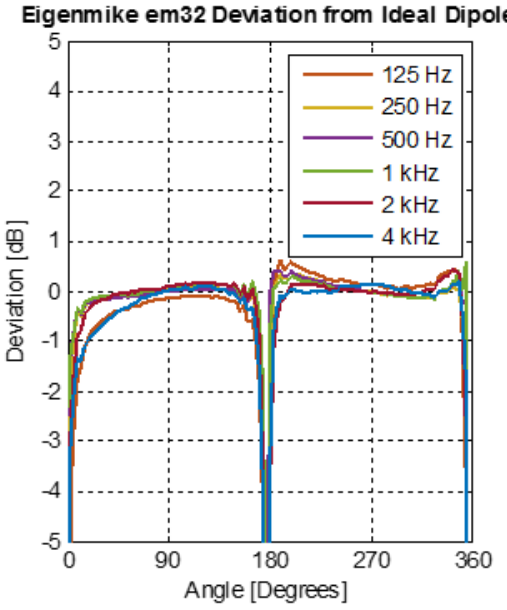

(b)

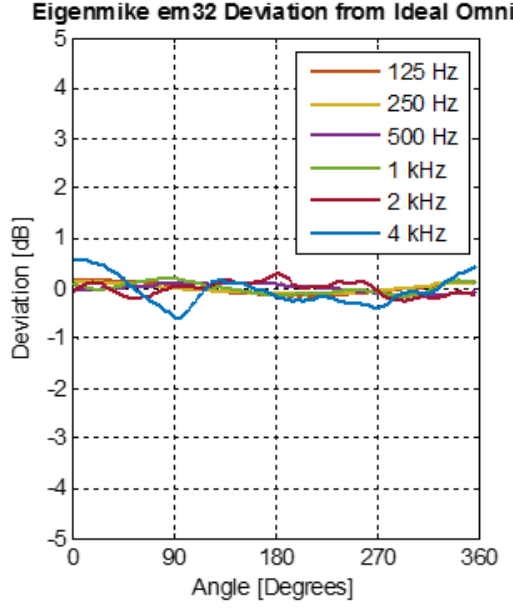

(c)

Figure 4: Deviations from ideal polar patterns: Sennheiser MKH30 figure-8 microphone directivity versus a perfect dipole (a), Eigenmike em 32 beamformed dipole versus a perfect dipole (b) and Eigenmike em 32 beamformed omnidirectional pattern versus a perfect omnidirectional (c) pattern.

\section{$292 \quad 4.3$ Impulse Response Measurements in Eisenhower Auditorium}

IR measurements were taken in the Eisenhower Auditorium (2500 seats) located on The Pennsylvania State University campus in University Park, PA, USA. The auditorium is a multipurpose venue used for a wide range of performance types and lectures. The measurements were taken with a moveable orchestra 
shell and overhead reflectors in place. The dodecahedron omnidirectional source was placed in the center of the stage for all measurements. Six receiver locations were chosen in the hall as shown in Figure 5: two on the main floor (R1 and R2), two on the grand tier level (R3 and R4), and two on the balcony level (R5 and R6).

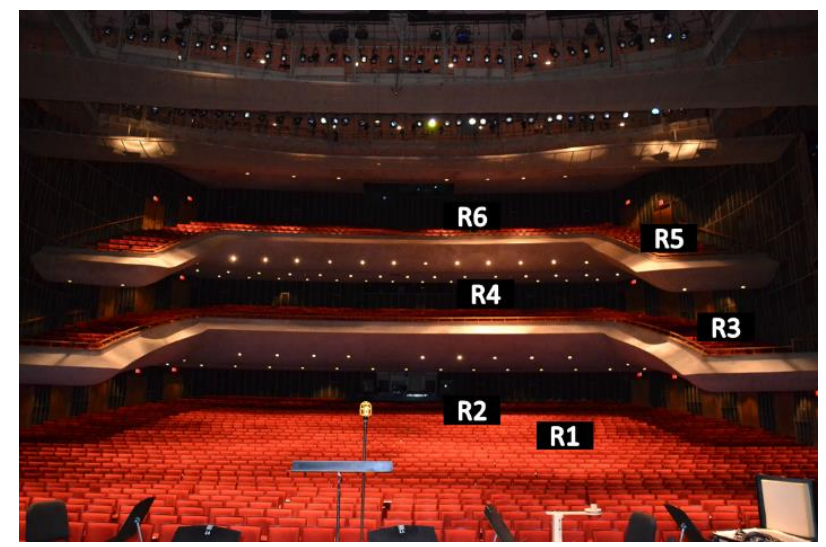

Figure 5: Receiver positions in 2500-seat Eisenhower Auditorium.

At each receiver position, the IR was obtained using the 2-channel FFT correlation measurement technique as implemented in the software program EASERA [32]. The excitation signal was a sine sweep with a pink-weighted spectrum. Each measurement was taken using 10 sweep averages, along with an additional pre-sweep to avoid audio artifacts. Measurements were taken using both microphone setups: the Eigenmike array, and the omnidirectional and figure-8 microphone pair. The center of each microphone setup (either the center of the Eigenmike array or the center of the two discrete microphones) was placed $20 \mathrm{~cm}$ from the seat back, $70 \mathrm{~cm}$ above the seat bottom, and halfway across the width of the chair, which were either 46 or $48 \mathrm{~cm}$ depending on the location in the hall. The Eigenmike array was aligned so that the 0 degrees azimuth direction was aligned with the source. The omnidirectional and figure- 8 pair was aligned such that the null plane of the figure- 8 microphone was oriented vertically toward the loudspeaker. The microphone alignments were done by eye. To evaluate measurement repeatability, a total of three measurement sets were taken for each microphone setup at each receiver location. In between sets, the custom microphone stand was removed and the various adjustment points were loosened and randomly repositioned. The stand was then replaced in the receiver location and re-aligned toward the source to re-measure the IRs for each microphone.

\section{DATA PROCESSING}

\subsection{Microphone frequency response compensation}

The frequency response of the omnidirectional sound source, the Sennheiser MKH30 microphone, and the Eigenmike array capsules were not found to have flat frequency responses, and thus required the application of equalization filters.

The Eigenmike random incidence frequency response was measured by playing uncorrelated pink noise over a nearly spherical loudspeaker array consisting of 30 loudspeakers in an anechoic chamber with the Eigenmike placed in the center of the array [33]. The monopole component of the Eigenmike measurement was extracted using Eqn. 9 and was then third-octave smoothed in the frequency domain. The same noise signal was measured using a B\&K 4942 diffuse-field $12.7 \mathrm{~mm}$ ( 0.5 inch) omnidirectional microphone placed in the center of the array to use as a reference, and then this signal was also one- 
third-octave smoothed in the frequency domain. The desired response for the Eigenmike equalization filter was generated by: (1) taking the smoothed spectrum of the B\&K microphone measurement and dividing by the smoothed spectrum of the Eigenmike monopole measurement, and (2) creating a 4096 point linear-phase FIR filter from the desired response using the MATLAB function "fir2" [34]. The filter was normalized so that the equalization applied unity gain at $1 \mathrm{kHz}$. This normalization convention allows for the equalization filter to be used after a single-tone $1 \mathrm{kHz}$ calibration. The magnitude response of the Eigenmike filter along with the target filter response are shown in Figure 6 as the solid and dashed blue lines, respectively.

Although the frequency response of the Sennheiser MKH30 is reasonably flat at mid-band frequencies, comparing the response of the MKH30 to the response of the $\mathrm{B} \& \mathrm{~K}$ measurement microphone or the Eigenmike revealed that the response deviates by $\pm 2 \mathrm{~dB}$ at high frequencies, and the response begins to gently roll off at low frequencies below $200 \mathrm{~Hz}$. An equalization filter was generated for the MKH30 using the anechoic chamber measurements described in Section 4.2. The desired filter response was generated by taking the one-third octave band smoothed spectrum of the free-field measurement of the MKH 30 directly on-axis, and dividing by the third-octave smoothed spectrum of the Eigenmike beamformed dipole on-axis with the Eigenmike's equalization filter applied as shown in Figure 6 as the dashed red line. A 4096 point linear-phase filter was generated using the same MATLAB function "fir2" as before, shown in Figure 6 as the solid red line. The on-axis free field measurements were used to equalize this microphone instead of diffuse field measurements.

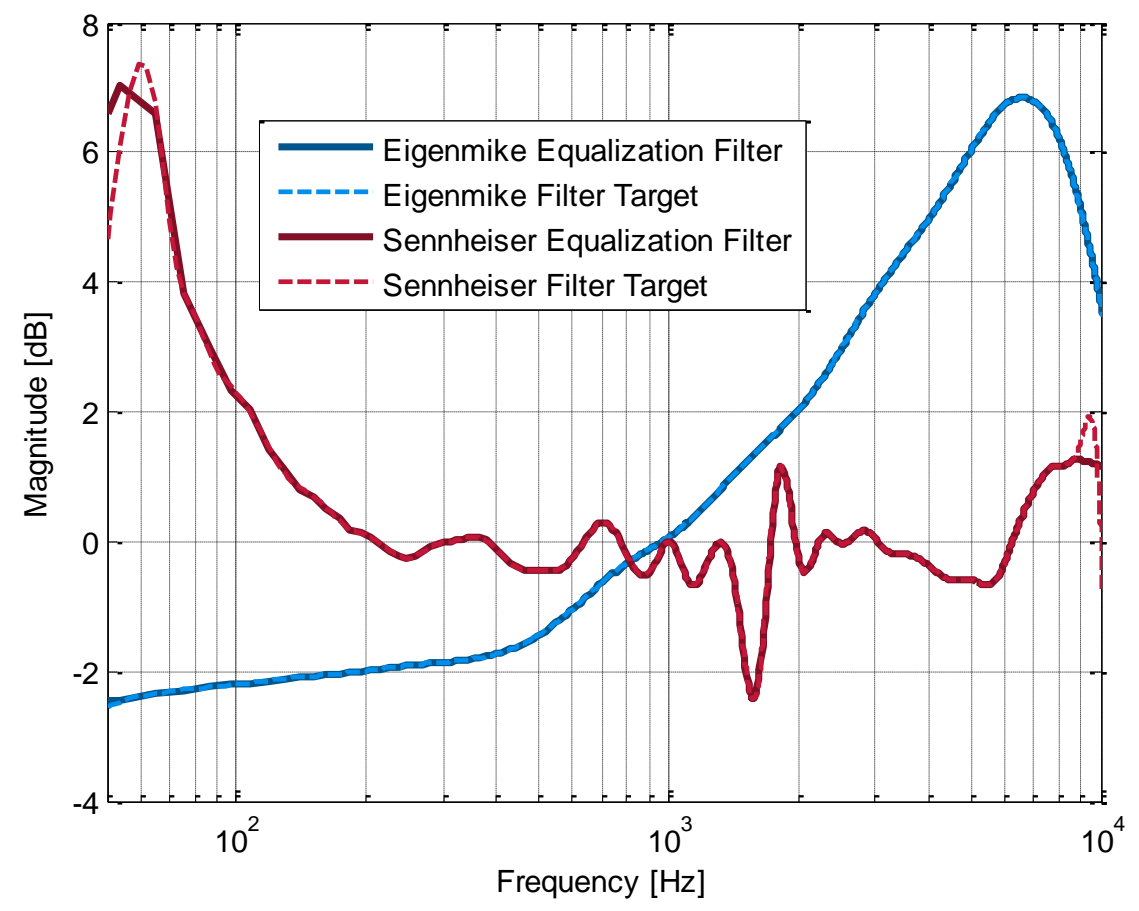

Figure 6: Eigenmike (blue) and Sennheiser MKH3O (red) equalization filter magnitude response. Target responses are shown as dashed lines, and realized filters fit to the target responses are shown as solid lines. The Eigenmike target and actual filter are nearly identical, which is why the dashed line is difficult to see in the figure. 


\subsection{Rotating the dipole}

For the figure-8 IR, the null plane should be pointed directly at the source. Alignments of the microphones were done by eye for this study. Alignment for the receiver locations toward the back of the hall and on upper seating levels can be especially difficult due to the source-receiver distances being the order of 35 $\mathrm{m}$. To correct for any rotational misalignments in the spherical microphone array measurements, the direct sound can be beamformed to determine the actual source direction (or the desired look direction), and the dipole beam can be steered to properly align the null plane.

For each Eigenmike IR, a rectangular window was applied in the time domain to extract only the direct sound from the IR, and a third-order plane wave decomposition was conducted using Eqn. 8 for azimuthal look directions from $-90^{\circ}$ to $90^{\circ}$ at $2 \mathrm{kHz}$. The $2 \mathrm{kHz}$ frequency bin was chosen because the inverse of the plane wave modal coefficients (Eqn. 5) are relatively small for the third-order coefficient at that frequency, and the high orders do not require a large gain. The angle with the maximum energy was found and that angle was used to rotate the dipole beam in azimuth.

\subsection{Room acoustic metric calculation}

In order to compare the measured IRs from the spherical array and the omnidirectional and figure-8 pair, the following metrics were evaluated: reverberation time (T30), early decay time (EDT), clarity index (C80), strength $(G)$, early lateral energy fraction $\left(\mathrm{J}_{\mathrm{LF}}\right)$ and late lateral energy level $\left(\mathrm{L}_{\mathrm{J}}\right)$. For the measures using the spherical array, the omnidirectional (monopole) and figure-8 (dipole) IRs were extracted from the raw IRs using Eqns. 9 and 10, respectively.

The omnidirectional parameters (T30, EDT, C80, and G) were calculated from the IRs measured from each microphone configuration, i.e. the monopole component of the Eigenmike, and the omnidirectional microphone. $\mathrm{J}_{\mathrm{LF}}$ was calculated using the monopole and dipole components of the Eigenmike measurements, and the omnidirectional and figure-8 microphone pair. $L_{\jmath}$ was calculated using the dipole component of the Eigenmike measurements and the figure- 8 microphone, respectively, with both configurations using the same free-field measurement of the sound source described in Section 4.2.

The measured IRs were analyzed by calculating the metrics following ISO 3382 [7] using MATLAB [34]. First, each IR was filtered to equalize the frequency response of the microphones and measurement loudspeaker (see Section 5.1). It is important to note that for the energy parameters, time-windowing was performed before octave-band filtering as defined in the standard. The order of this processing ensures that the group delay of the octave-band filters does not affect the energy integration limits. The results are presented as differences in the metrics, and compared with the just noticeable difference (JND) of the metric.

\section{RESULTS}

\subsection{Measurement Repeatability of the Two Microphone Configurations}

In this study, the largest source of measurement uncertainty is due to precise and accurate microphone placement in the exact same location for each measurement. In order to quantify the uncertainty due to microphone placement for each metric calculated for both microphone configurations as described in Section 5.3, the standard deviation across the three repetitions for each measure at each receiver location was obtained. The standard deviations were then compared to the JND of the specific metric as listed in Section 2. 
391 The standard deviations of the three repetitions for the six metrics measured with both microphone 392 configurations for all receiver positions were calculated, and are shown in Figure 7. The thick-dashed red 393 lines on each plot represent the respective 1 JND for each metric. In particular, the average standard 394 deviations for each metric are as follows: 0.087 JNDs for T30, 0.19 JNDs for EDT, 0.085 JNDs for C80, 0.073 395 JNDs for G, 0.092 JNDs for $\mathrm{JFF}_{\mathrm{LF}}$, and $0.069 \mathrm{JNDs}$ for $\mathrm{L}_{\mathrm{J}}$. The differences were all found to be less than $1 \mathrm{JND}$, 396 with a single exception of an EDT data point at $4 \mathrm{kHz}$ at R3. Of the six metrics evaluated in this study, EDT 397 was found to have the highest standard deviations relative to $1 \mathrm{JND}$. This result is consistent with a prior 398 study [21] and appears to indicate that EDT is the most susceptible to small differences in microphone 399 placement since this metric is dependent on the early sound field. The largest standard deviations for EDT 400 were found in the $2 \mathrm{kHz}$ and $4 \mathrm{kHz}$ octave bands. This finding makes sense because at high frequencies 401 the small microphone misalignments can be large relative to a wavelength.

402 Since prior research has indicated that there is a large degree of uncertainty with spatial measures [21,23403 25], it was hypothesized that the same would be true of the data in this study. However, as shown in 404 Figure 7, the spatial measures were found to be as consistent as T30, C80 and G. 
Reverberation Time (T30) Standard Deviation

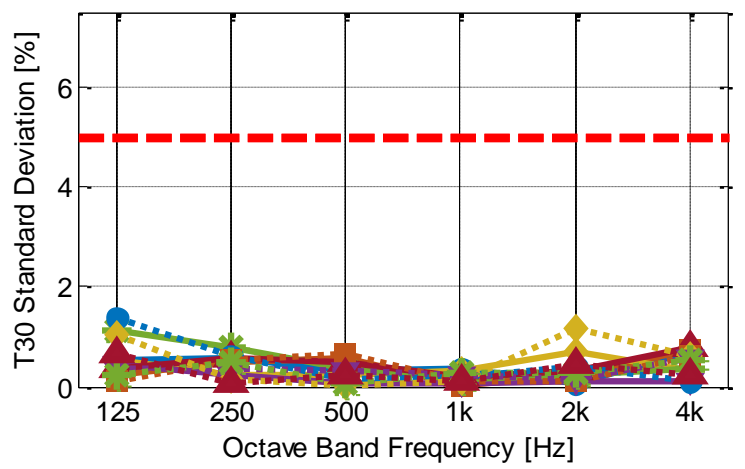

Clarity Index (C80) Standard Deviation

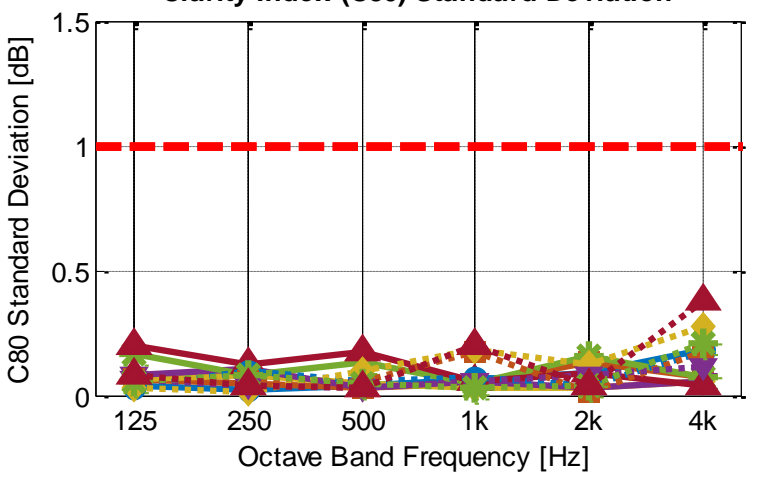

Lateral Energy Fraction $\left(\mathrm{J}_{\mathrm{LF}}\right)$ Standard Deviation
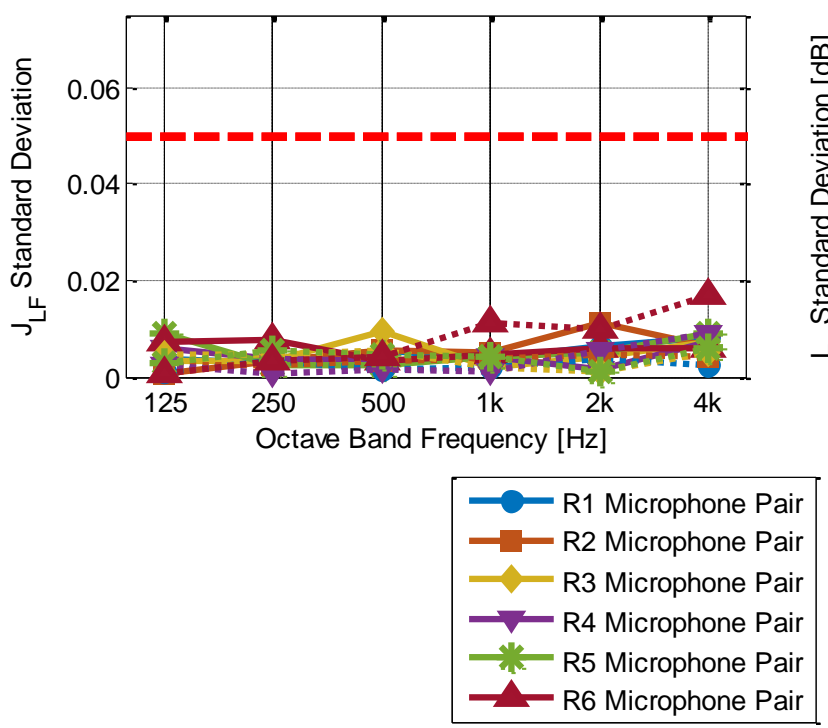

Early Decay Time (EDT) Standard Deviation
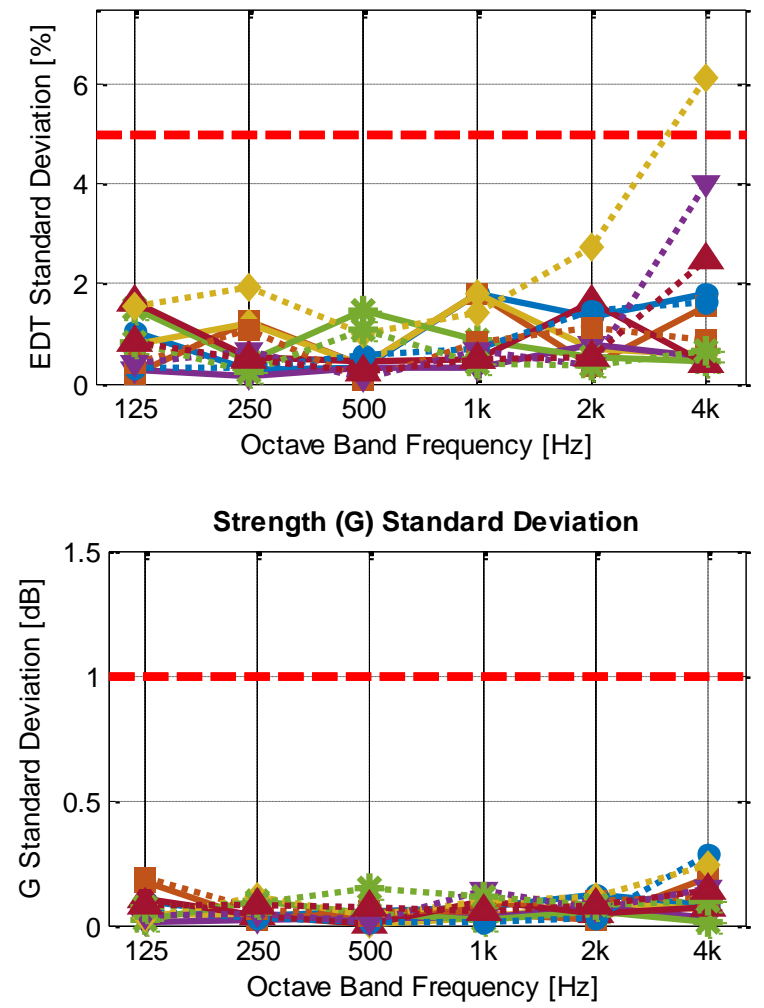

Late Lateral Energy Level $\left(L_{J}\right)$ Standard Deviation

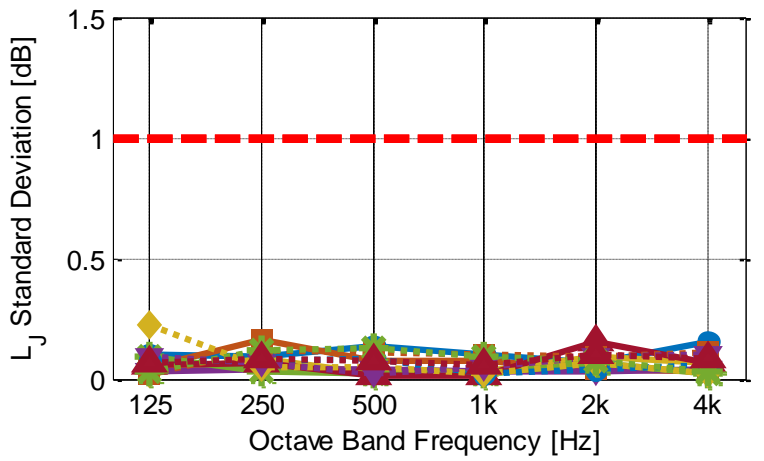

" - " R1 Eigenmike

" " - " R2 Eigenmike

" " " " " R3 Eigenmike

" - ". R4 Eigenmike

" " s." " R5 Eigenmike

". " R6 Eigenmike

Figure 7: Standard deviation of the three repeated measurements for each metric at each receiver location for the omnidirectional and figure-8 microphone pair (solid lines) and the Eigenmike array (thin-dashed lines) configurations. The thick-dashed red lines on each plot represent the respective 1 JND for each metric. All standard deviations were found to be well below 1 JND for each metric and all receiver positions, with the exception of a few cases for EDT. 
The measurement repeatability of the two microphone configurations were compared using the standard deviations of each calculated metric over the three measurements (solid versus thin-dashed lines in Figure 6). For $T 30, G, J_{L F}$, and $L$, the standard deviations were found to be similar with both microphone configurations. However, at high frequencies the two metrics that are the most susceptible to uncertainty due to microphone placement misalignments, EDT and C80, had larger standard deviations in the Eigenmike measurements. It was, in general, more difficult to align the Eigenmike in the physical space than traditional the traditional microphone pair, since the array is quite a bit larger than traditional measurement microphones and the round geometry makes it difficult to align the microphone's center or edges. The larger standard deviations confirm that the spherical array was more prone to slight alignment errors than the traditional microphones.

For the Eigenmike measurements, the dipole pattern was rotated so that the null plane was perfectly aligned with the direct sound. The authors hypothesized that the rotational alignment of the figure-8 microphone or the dipole pattern would be a significant source of uncertainty, which would result in a lower standard deviation for the spatial parameters $L_{J}$ and $J_{L F}$ measured with the Eigenmike than measured with the Sennheiser microphone. However, the standard deviations measured with the Eigenmike and the Sennheiser microphone do not appear to be appreciably different for the spatial parameters. Therefore, most of the variation is likely due to microphone placement and not due to rotational alignment. To further investigate the effect of a rotational misalignment, the Eigenmike IRs at R3 were beamformed with the null plane oriented in azimuth angles from -5 to +5 degrees in 0.1 degree steps, where the direct sound was incident at 0 degrees. The 5 degree misalignment caused an error in $\mathrm{J}_{\mathrm{LF}}$ of up to 0.02 , and an error in $L_{\jmath}$ of up to $0.15 \mathrm{~dB}$, which are both within $1 \mathrm{JND}$.

In addition to evaluating repeatability using standard deviations, a test-retest reliability analysis [35] was performed using the statistical software SAS [36]. For each microphone configuration, metric, and octave band, a correlation analysis was run between the three measurement repetitions over the six receiver locations. The measure of test-retest reliability is Cronbach's alpha coefficient, where the coefficient is a value between 0 and 1 and a higher value indicates more reliability. In general, a value of 0.7 or 0.8 indicates a high degree of reliability [35]. The majority of the 72 output coefficients were above 0.99 . The two data points that are below 0.99 are: for EDT at $4 \mathrm{kHz}$ measured with the Eigenmike (0.98), and T30 at $4 \mathrm{kHz}$ measured with the B\&K microphone (0.93). The results of this statistical test provide more evidence that the measurements are repeatable.

441 In summary, the overall measurement uncertainty was found to be less than 1 JND for all six metrics for 442 both microphone configurations. Given the low amount of uncertainty and the consistent results across 443 both microphone configurations, the following analysis, described in the next section, was carried out to 444 compare the calculated metrics based on the IRs of the two microphone types. This analysis was conducted to determine if a spherical array can be used instead of the traditional microphone pair to measure typical room acoustics metrics and is described in the next section.

\subsection{Differences in Measured Room Acoustics Metrics between Microphone Configurations}

To compare the measurements obtained using both microphone configurations as described in Section 5.3, the calculated metrics for each receiver position were averaged over the three repetitions. These averages were then directly compared between the two configurations by subtracting the metrics obtained using the spherical microphone configuration from the measurements taken using the traditional microphone configuration. These differences were compared to the JNDs for each metric. 
A plot of the differences for each parameter is shown in Figure 8. The differences in T30 and EDT are shown as percentages and the remaining metrics are shown as absolute differences in order to be able to compare measured differences to their respective JNDs, indicated by a thick-dashed red line. The average differences for all of the measures are as follows: 0.18 JNDs for T30, 0.71 JNDs for EDT, 0.27 JNDs for C80, 0.25 JNDs for $G, 0.28$ JNDs for $J_{L F}$, and 0.26 JNDs for $L_{J}$. The differences across the microphone configurations for all six metrics for all six receiver locations are within 1 JND with the exceptions of R3 at $2 \mathrm{kHz}$ for EDT and C80, and a few other data points for EDT, which are discussed below.

Of particular interest for this study were the differences in the spatial measures, $\mathrm{J}_{\mathrm{LF}}$ and $\mathrm{L}_{\mathrm{J}}$, obtained from the two microphone configurations considering previous studies showed a large amount of uncertainty across different microphones and different measurement teams [21, 23, 24, 25]. The results of this study show that in all cases the differences between the spherical array measurements and traditional measurements are within 1 JND for the spatial measures. These results indicate that the metrics obtained from the IRs of either microphone configuration are perceptually identical, and that the spherical microphone array will yield similar results to measurements made with a traditional microphone configuration.

To reduce the differences in the spatial measures $\mathrm{J}_{\mathrm{LF}}$ and $\mathrm{L}_{\mathrm{J}}$, the beamformed Eigenmike dipole was rotated in an attempt to match any rotational misalignments that may have been present in the Sennheiser figure8 measurements. The agreement between the two microphone configurations did not significantly improve after rotating the Eigenmike dipole in azimuth angles from -5 to +5 degrees in 0.1 degree steps. In fact, the variation decreased in some octave bands, but increased in other octave bands, which indicates that the rotational misalignments in these measurements are not the dominant source of the differences between microphone configurations. 
Early Decay Time (EDT) Difference
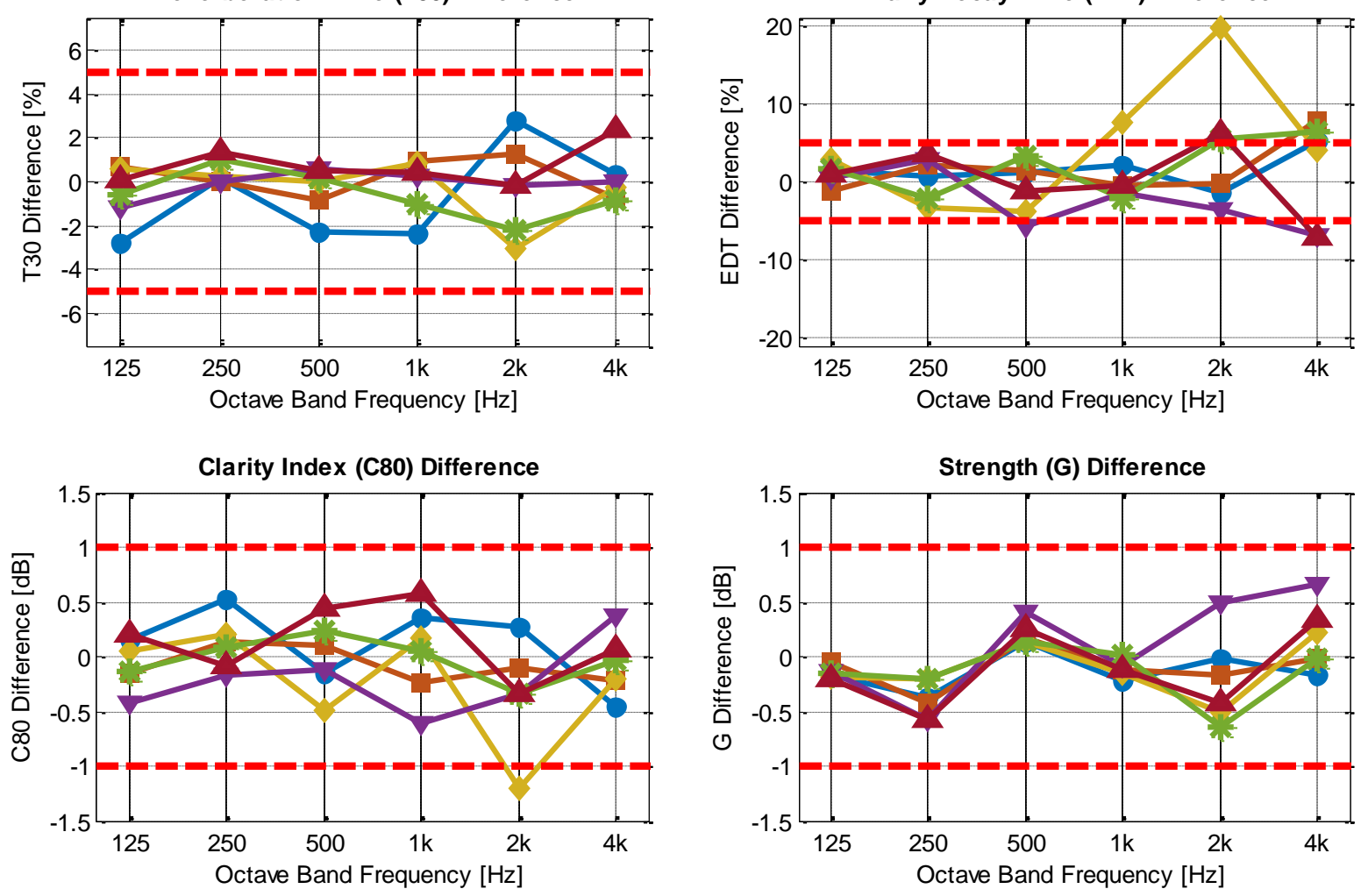

Lateral Energy Fraction $\left(\mathrm{J}_{\mathrm{LF}}\right)$ Difference


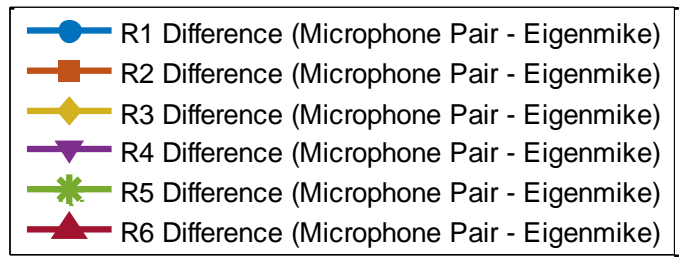

Figure 8: Differences between the omnidirectional and figure-8 microphone pair and the Eigenmike array configurations for all six metrics measured at all six receiver locations. The thick-dashed red lines on each plot represent the respective 1 JND for each metric. All differences were found to be within 1 JND, with the exceptions of a single point in C80, and several points in EDT at high frequencies. 
Although the differences between the two microphone configurations for most metrics are within 1 JND, 8 of the 216 data points (3.7\%) do exceed this threshold. Most of the differences were only slightly more than the $1 \mathrm{JND}$ threshold. In the $2 \mathrm{kHz}$ octave band, these differences were found in $\mathrm{C}_{80}$ at R3 and EDT and R6. In the $4 \mathrm{kHz}$ octave band, EDT differences slightly more than 1 JND were found at R2, R4, R5, and R6.

The single data point with a large discrepancy is the 4 JND difference in EDT in the $2 \mathrm{kHz}$ octave band at R3. A more detailed analysis of the measured IRs from the two microphone configurations at R3 was conducted to better understand this result. As shown in Figure 9, the differences in EDT can be seen by comparing the energy decay curves of the two microphone configurations. The decay curves are both normalized to a maximum level of $0 \mathrm{~dB}$. The slopes of the first $10 \mathrm{~dB}$ of the decay curves are very different primarily due to two spikes in the curves, as highlighted with ovals in Figure 9. These spikes may be a result of the physical location of R3 in the side portion of the first balcony (see Figure 5). This location is especially close to reflecting surfaces, including the side wall and the balcony ceiling. Since EDT is strongly influenced by early reflections, this measure may be particularly sensitive to the precise microphone position at this receiver location.

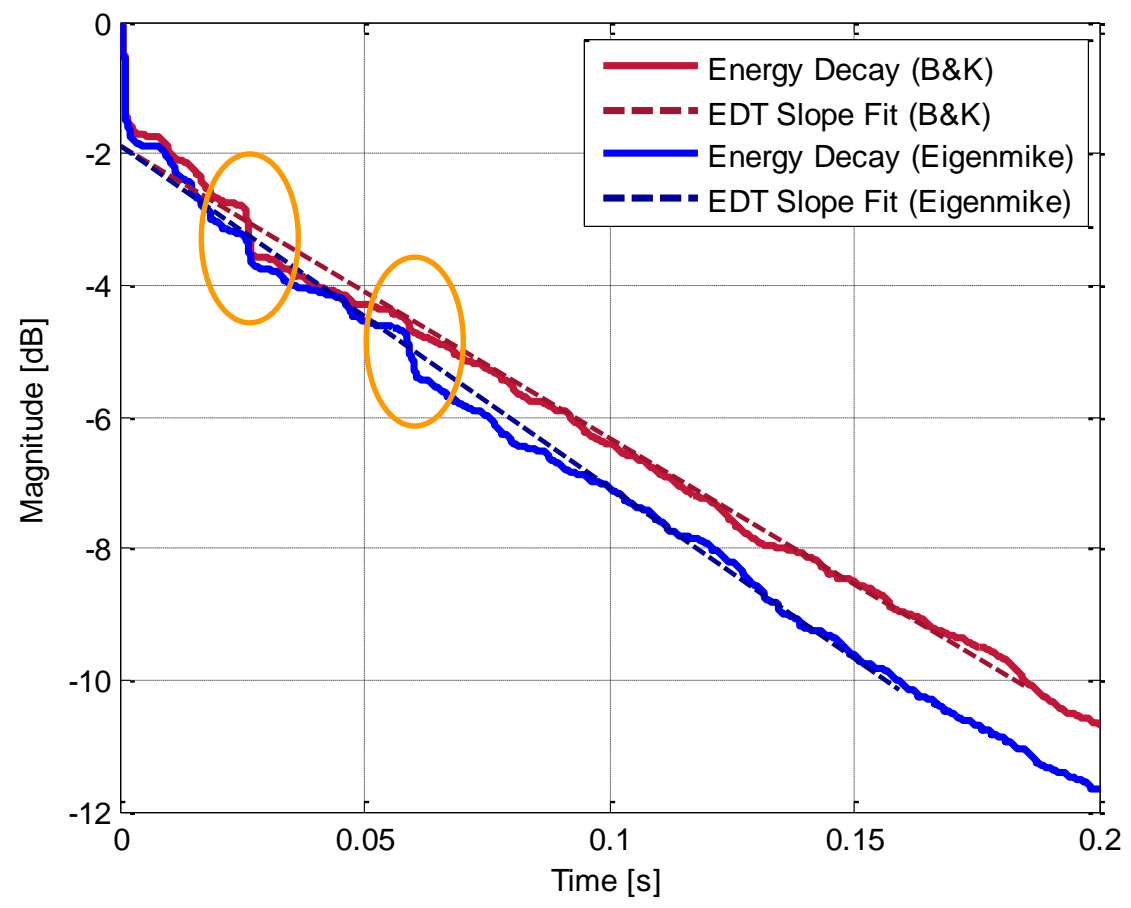

Figure 9: The energy decay curves and associated early decay time slope fits for $R 3$ in the $2 \mathrm{kHz}$ octave band shown for the omnidirectional B\&K microphone (blue) and Eigenmike array (red). Note the spikes in the curve as denoted with the orange ovals, which are likely due to differences in where the centers of each microphone were positioned.

One possible reason for the differences in EDT, and to a lesser extent C80, is that the center of (in other words the space between) the B\&K and Sennheiser pair was in the same position as the center of the Eigenmike array. As a result, the physical location of the center of the B\&K microphone was $3.8 \mathrm{~cm}$ in front of the center of the Eigenmike array. Although this location is still inside the 8.4-cm sphere that the Eigenmike array physically occupies, the measurement of EDT could vary significantly over the sphere of the array. To investigate this possibility, the EDT was calculated for each of the 32 individual omnidirectional Eigenmike capsules separately in the 125 to $4,000 \mathrm{~Hz}$ octave bands at R3, as shown in 
Figure 10. The variation in EDT across the 32 capsules exceeded the $5 \%$ JND in all octave bands. It was expected that the EDT values would have large variations across the 32 individual microphones at high frequencies due to the shadowing effect of the rigid sphere. However, in the $125 \mathrm{~Hz}$ and $250 \mathrm{~Hz}$ octave bands where the shadowing effect is negligible (i.e. very little energy is scattered from the 8.4-cm sphere), the range in measured EDT values still exceeds the 5\% JND. This finding provides more evidence that the EDT measurement is very sensitive to the physical microphone position. Given that the EDT values in all six octave bands measured with the B\&K microphone all fall within the range of EDT values measured with the individual Eigenmike capsules, the differences in measured values between the Eigenmike and $B \& K$ microphone are most likely caused by the offset of $3.8 \mathrm{~cm}$ in the position of the center of the microphones.

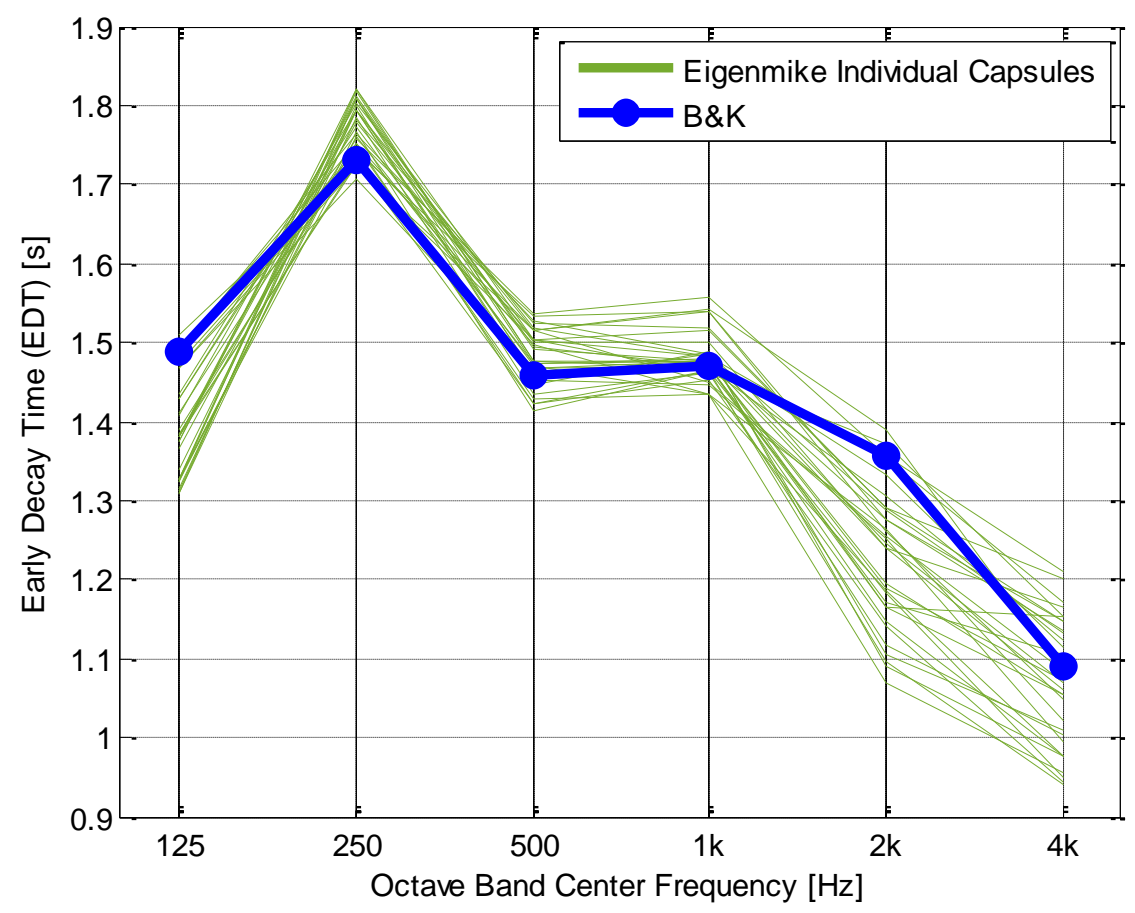

Figure 10: Early decay time calculated for Eigenmike individual microphone capsules (green), and early decay time calculated for the omnidirectional B\&K microphone (blue) at R3.

\section{CONCLUSIONS}

The purpose of this study was to compare the room acoustics metrics defined in ISO 3382 [7] when obtained from IRs measured with a conventional microphone configuration, an omnidirectional and figure-8 pair, to those measured with a spherical microphone array. IR measurements were obtained in a 2500-seat auditorium using a B\&K 419212.7 mm (0.5 inch) microphone and a Sennheiser MKH30 microphone, and an Eigenmike em32 spherical microphone array. Six receiver locations were measured throughout the hall, with three repetitions at each receiver to evaluate the uncertainty in the positioning of the microphones at each location. A custom microphone stand was utilized to ensure accurate and precise placement for each repetition. The room IRs were filtered to equalize the frequency response of the microphones and sound source, and metrics were extracted from the IRs using MATLAB. The measures evaluated as part of this study were reverberation time (T30), early decay time (EDT), clarity index (C80), 530 strength $(G)$, early lateral energy fraction $\left(\mathrm{J}_{\mathrm{LF}}\right)$, and late lateral energy level $\left(\mathrm{L}_{\mathrm{J}}\right)$. 
In order to evaluate measurement repeatability, the standard deviations of the three repetitions were calculated for each metric at each receiver location for both microphone configurations. The standard deviations were evaluated across the metrics and microphone configurations, and compared to the JND of each measure as defined in the Annex of ISO 3382 [7]. On average, across all metrics in the octave bands from 125 to $4000 \mathrm{~Hz}$ at all six receiver locations, the average standard deviations were 0.099 JNDs. One exception was found for a single standard deviation of $1.2 \mathrm{JNDs}$ for EDT in the $4 \mathrm{kHz}$ octave band at R3 measured with the Eigenmike. The deviation likely occurred due to slight misalignments in repositioning the spherical array during repetitions. A test-retest reliability analysis was performed, and Cronbach's alpha coefficient was found to be above 0.99 for the majority of the measurements (above 0.8 is considered to be the criteria for reliability), confirming that the three repetitions were consistent. Overall, microphone placement was found to be repeatable between the three repetitions of each measurement, which indicates that the measurement uncertainty is relatively low, and it is valid to compare the measurements made with each microphone configuration.

Differences in the four omnidirectional and two spatial metrics evaluated in this study, as obtained from the IRs between the two microphone configurations, were calculated at each receiver location. The differences in the measures were smaller than 1 JND for the majority of the metrics, with the exception of $\mathrm{C} 80$ at one single receiver position and octave band, and EDT in several receiver positions at high frequencies ( $2 \mathrm{kHz}$ and above). These metrics were found to be very sensitive to small changes in position of the microphones since they are dependent on the early sound field. The measurement differences in EDT and C80 are likely due to the physical center of the B\&K omnidirectional microphone being $3.8 \mathrm{~cm}$ in front of the physical center of the Eigenmike array, which was done in order to keep the center of the microphone pair and the center of the Eigenmike array coincident. Based on previous research, the authors' hypothesis was that the largest variation between microphone configurations would be found for the spatial metrics $L_{\jmath}$ and $J_{L F}$, but instead these metrics were found to have smaller differences than EDT and C80 relative to 1 JND.

Overall, the agreement in the calculated metrics obtained from the IRs of the two microphone configurations is much better than the agreement reported in previous studies for differing microphone types $[23,24,25]$. The measurement consistency in the results between the two microphone types was achieved because of matching directivity patterns between the microphones and beamformed signals, a flat frequency response for each microphone achieved by filtering to equalize the microphone signals, and accurate and precise placement of the microphones in the seat locations. Further studies are encouraged that include a larger number of microphones. Based on the results of this case study, it is acceptable to measure room acoustic metrics with a spherical microphone in place of the traditional configuration of an omnidirectional and figure-8 microphone pair. This finding will enable the use of spherical microphone arrays to measure both existing room acoustics metrics and to develop new metrics using higher order beamformed directivities.

\section{ACKNOLWEDGEMENTS}

The authors wish to express their thanks to Mr. Tom Hesketh, for allowing access to Eisenhower Auditorium for taking the measurements. The authors also wish to acknowledge Matthew Neal, Matthew Kamrath, Martin Lawless, and Acadia Kocher for their assistance with the measurements; and Matthew Neal for demonstrating the procedure used to position the microphone using the custom microphone stand in the online video [31]. The authors would like to thank Bose Corporation in Framingham, MA, USA 
2

3

4573 for use of their anechoic chamber to measure the directivities of the Sennheiser MKH30 microphone and

574 Eigenmike array. This work was supported by National Science Foundation (NSF) award \#1302741.

$\begin{array}{ll}7 & 575\end{array}$ 
[1] B. Rafaely, Fundamentals of spherical array processing, Berlin: Springer-Verlag, 2015.

[2] J. Meyer and G. Elko, "A highly scalable spherical microphone array based on an orthonormal decomposition of the soundfield," in Proc. IEEE Int. Conf. on Acoust., Speech, and Signal Proc., 2002.

[3] B. Gover, J. Ryan and M. Stinson, "Measurements of directional properties of reverberant sound fields in rooms using a spherical microphone array," J. Acoust. Soc. Am., vol. 116, no. 4, pp. 21382418, 2004.

[4] H. Sun, E. Mabande, K. Kowalczyk and W. Kellermann, "Localization of distinct reflections in rooms using spherical microphone array eigenbeam processing," J. Acoust. Soc. Am., vol. 131, no. 4, pp. 2828-2840, 2012.

[5] D. Khaykin and B. Rafaely, "Acoustic analysis by spherical microphone array processing of room impulse responses," J. Acoust. Soc. Am., vol. 132, no. 1, pp. 261-270, 2012.

[6] S. Clapp, A. Guthrie, J. Braasch and N. Xiang, "Three-dimensional spatial analysis of concert and recital halls with a spherical microphone array," in Proc. Int. Cong. on Acoust., Montreal, Canada, 2013.

[7] ISO 3382-1:2009: Acoustics - Measurement of room acoustic parameters - Part 1: Performance spaces.

[8] M. Barron, "Subjective effects of first reflections in concert halls - the need for lateral reflections," J. Sound \& Vib., vol. 15, no. 4, pp. 475-494, 1971.

[9] M. Barron, "Subjective Study of British Symphony Concert Halls," Acustica, vol. 66, no. 1, 1988.

[10] T. Lokki, J. Patynen, A. Kuusinen and S. Tervo, "Disentangling preference ratings of concert hall acoustics using subjective sensory profiles," J. Acoust. Soc. Am., vol. 132, no. 5, pp. 3148-3161, 2012.

[11] J. S. Bradley and G. A. Soulodre, "The influence of late arriving energy on spatial impression," J. Acoust. Soc. Am., vol. 98, no. 5, pp. 2263-2271, 1995.

[12] J. S. Bradley and G. A. Soulodre, "Objective measures of listener envelopment," J. Acoust. Soc. Am., vol. 81, no. 4, pp. 344-355, 1995.

[13] L. Beranek, Concert Halls and Opera Houses: Music, Acoustics, and Architecture, New York: Springer, 2003.

[14] M. Barron and A. H. Marshall, "Spatial Impression Due To Early Lateral Reflections in Concert Halls: The Derivation of a Physical Measure," J. Sound \& Vib., vol. 77, no. 2, pp. 211-232, 1981. 
[15] I. B. Witew and P. Dietrich, "Assessment of the uncertainty in room acoustical measurements," in Proc. Int. Cong. on Acoust., Madrid, Spain, 2007.

[16] R. San Martín, "Uncertainties caused by source directivity in room-acoustic investigations," J. Acoust. Soc. Am., vol. 123, no. 6, pp. EL133-EL138, 2008.

[17] R. San Martín, I. Witew, M. Arana and M. Vorländer, "Influence of the source orientation on the measurement of acoustic parameters," Acta Acust. united Acust., vol. 93, no. 3, pp. 387-397, 2007.

[18] D. de Vries, E. M. Hulsebos and J. Baan, "Spatial fluctuations in measures for spaciousness," J. Acoust. Soc. Am., vol. 110, no. 2, pp. 947-954, 2001.

[19] R. San Martin, I. Ganuza, M. Arana, A. Vela, M. San Martín, E. Aramendía, S. Galech and V. Latorre, "Influence of the Listener Position on the Measurement of Acoustic Parameters," in Proc. Int. Congr. Noise Control Eng., INTERNOISE, Rio de Janeiro, Brazil, 2005.

[20] A. Lundeby, T. Vigran, H. Bietz and M. Vorländer, "Uncertainties of measurements in room acoustics," Acustica, vol. 81, no. 4, pp. 344-355, 1995.

[21] I. Bork, "Report on the 3rd Round Robin on Room Acoustical Computer Simulation - Part I: Measurements," Acta Acust. united Acust., vol. 91, no. 4, pp. 740-752, 2005.

[22] P. Fausti and A. Farina, "Acoustic measurements in opera houses: Comparison between different techniques and equipment," J. Sound \& Vib., vol. 232, no. 1, pp. 213-229, 2000.

[23] S. Geroso, A. P. Belli, M. Giovannini and R. Spagnolo, "Measurement uncertainty of the reverberation time and others acoustical parameters changing model of microphone," in Proc. Euronoise, Tampere, Finland, 2006.

[24] I. Witew, G. K. Behler and M. Vorländer, "Spatial variation of lateral measures in different concert halls," in Proc. Int. Cong. on Acoust., Kyoto, Japan, 2004.

[25] M. C. Vigeant, C. B. Giacomoni and A. C. Scherma, "Repeatability of spatial measures using figureof-eight microphones," Appl. Acoust., vol. 74, no. 9, pp. 1076-1084, 2013.

[26] E. G. Williams, Fourier Acoustics: Sound Radiation and Nearfield Acoustical Holography, New York: Academic Press, 1999.

[27] B. Rafaely, "Plane-wave decomposition of the sound field on a sphere by spherical convolution," J. Acoust. Soc. Am., vol. 116, no. 4, pp. 2149-2457, 2004.

[28] C. T. Jin, N. Epain and A. Parthy, "Design, Optimization and Evaluation of a Dual-Radius Spherical Microphone Array," IEEE Trans. Audio Speech Lang. Process. , vol. 22, no. 1, pp. 193-204, 2014.

[29] S. Tervo and A. Politis, "Direction of Arrival Estimation of Reflections from Room Impulse Responses using a Spherical Microphone Array," IEEE/ACM Trans. Audio Speech Lang. Process., vol. 23, no. 10, pp. 1539-1551, 2015. 
[30] "em32 Eigenmike microphone array release notes (v17.0)," mh acoustics, 30 October 2013. [Online]. Available: http://www.mhacoustics.com/sites/default/files/ReleaseNotes.pdf. [Accessed 27 August 2015].

[31] D. A. Dick, M. T. Neal and M. C. Vigeant, "SPRAL Custom Microphone Stand Video," [Online]. Available: http://sites.psu.edu/spral/equipment/.

[32] "EASERA v 1.2," AFMG TechnologiesGmbH, [Online]. Available: http://easera.afmg.eu/. [Accessed 27 August 2015].

[33] M. Neal, "Investigating the sense of listener envelopment in concert halls using a third-order ambisonic reproduction over a loudspeaker array and a hybrid room acoustics simulation method," M.S. Thesis, Penn State University, 2015.

[34] "MATLAB," Mathworks, [Online]. Available: http://www.mathworks.com/products/matlab/. [Accessed 27 August 2015].

[35] A. Field, Discovering Statistics using SPSS, Third Edition, Thousand Oaks, CA: SAGE Publications, 2009.

[36] "SAS 9.4 for Windows," SAS Institute, [Online]. Available: www.sas.com. [Accessed 2 September 2015]. 